\title{
LA UNIVERSIDAD EN LA SOCIEDAD DEL CONOCIMIENTO: HACIA UN MODELO DE PRODUCCIÓN Y TRANSFERENCIA DE CONOCIMIENTOS Y APRENDIZAJES
}

\author{
Axel Didriksson TaKayanagui*
}

Recebido: 3 jun. $2014 \quad$ Aprovado: 31 jul. 2014

* Universidad Nacional Autónoma de México (UNAM). México DF, México. E-mail: didrik@unam.mx

Resumen: En este trabajo se argumenta sobre la urgente transformación de las políticas públicas y universitarias, de la investigación científica y tecnológica, en el contexto de una sociedad del conocimiento, con el fin de propiciar procesos sustentables de cognición, que pongan en el centro el desarrollo de capacidades para la investigación desde el contexto de su aplicación, y que haga posible dar un salto de calidad para transitar del actual modelo de educación y de universidad Modo 1, de tipo disciplinar, profesionalizante, lineal, centrado en la difusión de un conocimiento reiterado y repetitivo, hacia uno en donde la multiplicación de medios de aprendizaje hagan posible la indagación y el aprendizaje que construye soluciones centrado en la producción de conocimientos.

Palabras clave: Cambio en la educación superior. Nueva universidad. Producción de conocimientos. Curriculum universitario. Innovación académica.

\section{THE UNIVERSITY IN A KNOWLEDGE SOCIETY: \\ TOWARD A MODEL OF TRANSFER OF KNOWLEDGE PRODUCTION AND LEARNING.}

Abstract: This paper argues the urgent transformation of public and university policies, scientific and technological research in the context of a knowledge society, in order to provide sustainable processes of cognition, that place at its center the development of research capacity, and that makes it possible to promote a qualitative leap to move from the current model of education and Mode 1 university, of the disciplinary type -- professional, linear, focused on dissemination of repeated and repeatable knowledge -- to one in which the multiplication of means of learning enable the inquiry and learning that builds solutions in knowledge production.

Key words: Change in higher education. New university. Knowledge production. University Curriculum. Academic Innovation.

\section{A UNIVERSIDADE NA SOCIEDADE DO CONHECIMENTO: RUMO A UM MODELO DE PRODUÇÃO E TRANSFERÊNCIA DE CONHECIMENTO E APRENDIZAGEM}

Resumo: Neste trabalho argumenta-se sobre a urgente transformação das políticas públicas e universitárias, da investigação científica e tecnológica, no contexto de uma sociedade do conhecimento, com o fim de propiciar processos sustentáveis de cognição, que ponham no centro o desenvolvimento de capacidades para a investigação a partir do contexto de aplicação, e que torne possível dar um salto de qualidade para transitar do atual modelo de educação e de universidade Modo 1, de tipo disciplinar, profissionalizante, linear, centrado na difusão de conhecimento reiterado e repetitivo, para um em que a multiplicação de meios de aprendizagem possibilitem a indagação e a aprendizagem que constrói soluções na produção de conhecimentos.

Palavras-chave: Mudanças na educação superior. Nova universidade. Produção de conhecimento. Currículo universitário. Inovação acadêmica. 
En este trabajo se argumenta sobre la urgente transformación de las políticas públicas (tanto al nivel del Estado como institucional) respecto de la universidad y la educación superior, de la investigación científica y tecnológica, de sus necesarias y pertinentes articulaciones, con el fin de propiciar procesos sustentables de cognición, que ponga en el centro el desarrollo de capacidades para la investigación desde el contexto de su aplicación, y que haga posible dar un salto de calidad que haga posible transitar del actual modelo de educación y de universidad Modo 1, hacia otro de Modo 2 (GIBBONS et al., 2007); de uno de tipo disciplinar, profesionalizante, lineal, centrado en la difusión de un conocimiento reiterado y repetitivo, hacia uno en donde la multiplicación de medios de aprendizaje hace posible la indagación, el aprendizaje que construye soluciones centrado en la producción de conocimientos, y supere el actual sistema "dual" de instituciones de educación superior organizado en procesos rígidos de enseñanza limitados al aula, verticales, enciclopédicos y memorísticos; concentrado en algunas carreras saturadas y con perfiles de egreso de escasa relación con los mercados laborales más dinámicos, entre otros de sus atributos que se generalizan y mantienen sin cambios desde hace décadas.

Se trata de proponer superar este tipo de institución que se ha reproducido en una suerte de polarización: unas relacionadas con la formación de elites, frente a otras que se masifican y se saturan, ambas sin la debida adecuación de sus estructuras, más allá de las modas que frecuentan y buscan imponer, de vez en vez.

Este modo de operar, esta organización y gestión de la de la curricula y de la enseñanza, refleja el actual sistema de desigualdades e inequidades sociales, económicas y culturales, desde una enseñanza que sirve como medio de dominación, de aculturación mercantil y consumismo individualizado, frente a los requerimientos de poder alcanzar un nuevo sistema de desarrollo más justo y equitativo, que forme para una ciudadanía activa, crítica y participativa, y que se sustente en una visión prospectiva de sociedad democrática del conocimiento (DIDRIKSSON, 2007).

El tema no es nuevo y existe un abundante trabajo de referencias de enfoques, definiciones y corrientes de pensamiento, ejemplos y experiencias de la manera como está ocurriendo un largo proceso de cambio en la universidad contemporánea, que va de la vigencia de un sistema educativo formal hacia otro de producción y transferencia de aprendizajes y conocimientos, con el desarrollo de plataformas sociales de capacidades para aprender a aprender e innovar, con objetivos de bienestar general. 
Los componentes modernos de un sistema de producción y transferencia de conocimientos y aprendizajes, se organiza desde la trans y la interdisciplina; y, los nuevos paradigmas del aprendizaje desde una multiplicidad de medios ambientes de educación formal y no formal, presenciales y a distancia, en una imbricación que da cuenta de las nuevas expresiones organizacionales que se están alcanzando en la gestión de las instituciones de educación superior y de investigación científica y tecnológica, con componentes de innovación social e intelectual que van de la investigación cognitiva y estratégica pegadas a la generalización de tecnológicas que hacen posible la creación de redes de aprendizaje y conocimiento, relacionados a la internet, a la virtualidad, a los nuevos materiales o a las nano-bio-ciencias, a las humanidades y a las nuevas perspectivas de la complejidad y la sociedad líquida, y desde lo que se puede estudiar en las inmensidades del espectro de los macrodatos (The Big Data) ${ }^{1}$.

En este nuevo deslave de pasados y circunstancias, en la liquidez en la que todo pasa y nada queda, se ha considerado que el impacto económico de las universidades y de los centros generadores de realidades virtuales e innovaciones sociales y tecnológicas, es tal que, se arguye, se está produciendo un cambio estructural en el mismo desarrollo económico y social, como un todo, sumergido como está, sin embargo, en el contexto del largo declive de la actual sociedad en riesgo (BRENNER, 2013), de crisis frecuentes y sostenidas, y rupturas en los marcos de sus relaciones globales y de dominación.

Como el conocimiento es cada vez más genérico y público, como el aire o la luz, este se ha convertido (como generador que en algún momento lo fue) en el primer componente de cuestionamiento de la actual globalidad diferenciada, una no sólo efímera y copiosa, sino también predadora. En los textos recientes de dos autores prominentes, Zygmunt Bauman (2008) y Daniel Innerarity (2012), se leen algunos de los códigos que a menudo aparecen como indescifrables para la ciudadanía global, porque se muestran como un vaso de efervescencia burbujeante que desaparece rápidamente para dar solución a esa pequeña acidez que llega de manera tan frecuente.

El conocimiento, se ha convertido en un poder de generación de ganancias inconmensurables para unos cuantos, pero también en el contenido, método, lenguaje y técnica de una enorme multiplicidad de procesos y productos cada vez más accesibles y cotidianos, que están avanzando en un camino distinto a

1 Véase, por ejemplo, Kenneth Cukier and Viktor Mayer-Schoenberger (2013). Diego Molano; José de la Peña Mario Tascón et al. (2013). 
la prefiguración de cualquier ortodoxia pasada, ya sea si se cree en el capitalismo de un nuevo tipo o si se está en su contra, aún de la forma más radical que se pueda uno imaginar.

El tema es por ello relevante, sobre todo cuando están en proceso revoluciones sociales mediadas por las tecnologías y las redes de comunicación existentes, que han surgido desde países y ciudades (desde el norte de Africa, pasando por Europa y los países árabes, hasta llegar a América Latina; ¿los nuevos eslabones de la ruptura neo-global?), en donde se entremezclan dominios individuales de grandes riquezas, con revueltas masivas de pobres, jóvenes e inmigrantes, mediadas por valores comunes que demandan educación, conocimiento y nuevos aprendizajes, con fines de igualdad y justicia.

El aprendizaje de determinados conocimientos por la vía de una educación sistemática y significativa, se ha vuelto un vínculo esencial para alcanzar niveles distintos de poder en este mundo de riesgos, en tendencias sociales bifurcadas en donde no se sabe bien a bien el tipo de capitalismo que se busca o el por qué se llevan a cabo sus contrastes, cuando aparecen de forma similar y multiplicada en distintos lugares, y luego tienden a desintegrarse.

En la mediación de todo este cambio general, están los conocimientos complejos organizados de forma convergente como nunca antes se había visto, y la expansión de sistemas de aprendizaje, la mutación de las instituciones que los generan -como las universidades y otras instituciones culturales y científicas-, y actores que siempre destacan en estos procesos: estudiantes, intelectuales, investigadores, profesores, bajo la forma de una nueva clase con identidades compartidas pero también divergentes de futuro, como la configuración (no) organizada de un nuevo tipo de sociedad civil de carácter red-virtual, relacionada con tecnologías cada vez más pegadas al cuerpo a través de las cuales se ejerce una nueva conciencia contestataria multitudinaria.

La curiosidad de la época, es que en unos cuantos años se agotó el esquema unidimensional de una economía globalizadora (entendida como un modelo unipolar e integrador, dominado por Estados Unidos), y se ha entrado de lleno a otro de carácter multipolar y diverso, en donde está destacando la irrupción de los pueblos del norte de Africa y del centro del mundo árabe como los más resistentes al esquema de un solo polo dominante (y no sólo por razones religiosas); otros países que están llevando a cabo modelos propios (como Corea, India, Singapur, China o Brasil), u otra serie de países -otrora denominados "desarrollados" o "subdesarrollados"-, que están relacionados con su destacado y específico papel en alguna de las partes de la nueva división internacional de los conocimientos y las tecnologías (Finlandia, Suecia, Noruega, Rusia, et 
al.), o bien por la reconfiguración de sus modos de trabajo y producción, sobre todo en Asia y en América Latina -con gobiernos no controlados por la otrora lógica hegemónica europea o norteamericana y hasta en abierto desafío hacia ésta, sobre todo en el terreno político y cultural-, y que, de manera particular, fueron quienes no se adaptaron ni siguieron de forma ortodoxa a los designios del denominado "consenso de Washington".

$\mathrm{Su}$ "emergencia" -o diferenciación- en la descomposición del actual mundo global está, sin embargo, amarrada a una cantidad desquiciante de imponderables, sobre todo por la constancia de los constantes vaivenes y rupturas financieras que se presentan de forma cíclica en los países ricos, por la agudización de la competitividad alrededor de los productos de frontera con mayor valor agregado en conocimientos y tecnología, y por la concentración monopólica que ejercen sobre ellos un puñado de transnacionales. En medio de todo esto está la dinámica diferenciada de los umbrales demográficos mundiales: el envejecimiento poblacional de Asia y Europa y el "boom" juvenil latinoamericano.

Estaca en este contexto de cambio lo que pueden jugar los gobiernos en turno, en esta transición prolongada (como paradoja, en un periodo en el que se buscó que los Estados podrían retirarse a sus tareas sociales y económicas, de logros de bienestar a favor de relaciones de mercado, porque en todo lo demás apenas y harían falta), ante el avance avasallador del capital financiero que requiere de su reactivación y concentración para poder pasar de sus sucesivas emergencias coyunturales $(2009 ; 2013 ; 2015-2016)$, para alcanzar su eventual nueva concentración de capitales y la recomposición de sus políticas de regulación de favorecimiento transnacional, en esta época en la que se frecuentan nuevos tipos de revoluciones: sociales, económicas, ecológicas, de género, multiculturales, tecnológicas, científicas, sub-urbanas..., que requieren y exigen poner en marcha nuevas formas de gobernabilidad democrática, a la altura de las exigencias de una sociedad cada vez más consciente, diversa y activa, sobre todo cuando ésta se enlaza, desde sus distintos ámbitos laborales y políticos con las dinámicas de la producción y transferencia de aprendizajes y conocimientos, y se envuelve, de forma cotidiana, en su quehacer mediado por múltiples objetos tecnológicos móviles.

Rebasadas, por el mercado del comercio del valor agregado en conocimientos y tecnologías, las universidades se han visto mucho más lentas en llevar a cabo cambios pertinentes al ritmo de lo que ocurre en la organización y gestión de nuevos conocimientos, porque estaban acostumbradas a llevarlos a cabo de forma contestataria, o a menudo de manera retardataria. 
No obstante, y a su pesar, la multiplicación de actividades de desarrollo científico y tecnológico está impulsando un fuerte impacto sobre las mismas, pero aun así sus respuestas ante las crisis sucesivas y las rupturas epistémicas en los mismos conocimientos se ha visto bastante diferenciada y fragmentada $y$, para el caso de las mexicanas, verdaderamente marginal.

Las evidencias muestran, entonces, que de ser instituciones críticas organizadas para preservar la cultura y reproducir disciplinas y profesiones, las universidades pasaron a ser organismos sociales con enormes demandas insatisfechas para producir "conocimientos sobre conocimientos", por la vía de aprendizajes y la investigación referenciada, o de Modo 2, y con ello dejaron pasar décadas de oportunidades para aprovechar las bondades de constituir su nuevo paradigma organizacional.

Contar con conocimientos organizados en instituciones de bien público, es central para la sustentación de cualquier plataforma de nuevo desarrollo social, porque hace referencia a "algo intangible" que no puede ser localizado sino en los métodos, lenguajes, habilidades, formas de aprender y en lo que se denomina de forma genérica como el ethos de la academia, visto como su aspecto "genérico".

Con la proliferación de redes de producción y transferencia de información y conocimientos, la expresividad de la relación entre docencia y aprendizajes ha alcanzado dimensiones extraordinarias, "en tiempo real" (frente a lo que antes hacía referencia a mecanismos epistolares, o a intercambios y convenios interinstitucionales), impulsando el cambio de las instituciones otrora educativas, hacia otras que producen, generan, recrean, difunden, transfieren y organizan aprendizajes y conocimientos.

Si la capacidad de este nuevo "ethos académico" no puede ser construido desde políticas institucionales y de Estado (dado que se trata de un bien común, de un valor social extraordinario y de un derecho fundamental) en la perspectiva de su sustentabilidad -esto es, para el largo plazo-, simple y llanamente no podrá ocurrir ninguna alternativa en la modificación del tipo de desarrollo vigente, hacia una perspectiva de bienestar integral para la población, la localidad, la región o el país.

2 "But there are some types of knowledge that are more difficult to codify and exchange in a market. There is "tacit" knowledge - skills which often cannot be reduced to mere information. Some human capabilities, such as intuition, insight, creativity and judgement, resist codification. And it is these tacit skills which are essential to selecting, using and manipulating the knowledge which can be codified. The ability to select relevant (and disregard irrelevant) information, to recognise patterns in information and to interpret and decode information is not easily bought and sold" (STEVENS, 1998, p. 90-91). 
Ubicadas como instituciones de gran trascendencia para fines de desarrollo económicos, social, cultural y de bienestar y solidez ciudadana, las expectativas que se ciernen sobre las universidades y la producción y transferencia de conocimientos que llevan a cabo están provocando presiones sin límite, la redefinición de políticas y planes, la frecuencia de programas y alternativas en la búsqueda y experimentación de modelos de organización e innovación.

Estas tendencias e impactos, que a lo largo de este trabajo se han analizado y documentado, hacen referencia a una dialéctica de escenarios que han alterado de forma significativa lo que la idea de universidad era hasta hace unas cuantas décadas, en relación a sus funciones, a sus sectores, a su gobernanza, a su calidad y a su lugar mismo en la sociedad.

Este escenario de nueva universidad, por el modelo de innovación que impulsa, estaría organizado para producir y transferir un alto valor social en conocimientos y aprendizajes, con la pertinencia y responsabilidad social en sus tareas y procesos académicos y de bien público de la institución hacia su entorno. La transformación de sus estructuras van tomando el cuerpo de una red que se sostiene en plataformas de cooperación interinstitucionales de todo tipo y nivel desde proyectos compartidos, con un muy amplia movilidad de académicos y estudiantes, con la co-participación de recursos, instalaciones y equipo y con una orientación educativa no punitiva sino sustentada en un aprendizaje múltiple, significativo, complejo, interdisciplinario e inter y transcultural. Los valores educativos se comparten y son motivo de análisis e investigación, la docencia se articula con la investigación cognitiva y se da un fuerte impulso a la investigación estratégica con el objetivo de que el cambio de contenidos sea permanente y en correspondencia con el logro de objetos de conocimiento y aprendizaje, la creación de nuevas habilidades y capacidades de resolución de problemas desde el contexto de su aplicación. Este modelo se articula con una amplia posibilidad de innovación, con actores comunitarios y regionales, nacionales e internacionales que la impulsan de adentro hacia afuera de la universidad.

Es por lo anterior, que el escenario de cambio y nueva reforma de la universidad, en el contexto de la transición larga en la que nos encontramos, se presenta como uno de tipo alternativo, porque pone el acento en la atención a las demandas contemporáneas de una sociedad desigual e inequitativa, en lugar de abstraerlas o hacer caso omiso de ellas, y porque pone el acento de la formación universitaria en la calidad entendida como el valor social de los conocimientos y aprendizajes que se producen y se transfieren desde una gestión institucional distinta, horizontal y cooperante. 
Esta concepción de nueva universidad, se sostiene en la construcción de una identidad que hace posible la fortaleza institucional enraizada en la problemática social, en la comprensión de la necesidad de impulsar un desarrollo original, en la búsqueda de reconstituir las capacidades de las personas como actores reales de su proceso cognitivo y en evitar que la educación siga siendo un mecanismo de desigualdad, de violencia, de competencia mercantil y de diferenciación racista.

Sobre todo esta concepción alternativa, supone pensar la calidad educativa distinta a la que se promueve de resultados, exámenes estándares y rankings, sino desde las condiciones en las que puede lograrse la transformación de las personas hacia una ciudadanía con valores de bienestar general, de confianza y de alta capacidad para mejorar la sociedad en la que vive y trabaja desde el valor social del conocimiento y del aprendizaje que demuestra en la práctica cotidiana.

En las experiencias que se tienen de desarrollos de cambios de modelo universitario, se muestra que el cambio parte desde los cuerpos directivos bajo contexto de alta exigencia social y comunitaria, y se pone en marcha bajo formas de "auto-regulación" y proceso de consensuamiento, que la formulación del proyecto tiene una visión de largo plazo y voluntades de altura para hacer participar a los actores centrales de los procesos educativos. El cambio propicia con ello estructuras más flexibles y dinámicas.

Ello supone que, para iniciar una reforma hacia la innovación desde las estructuras universitarias actuales, caracterizadas por su segmentación, burocratización y linealidad, poco articuladas a las demandas de la sociedad y poco enraizadas en los cambios de una economía que se transforma desde los conocimientos, la ciencia y la tecnología, se requiere sustentar nuevas estructuras organizacionales de tipo horizontal como catapultas para propiciar cambios pertinentes hacia el futuro.

Este punto de arranque debe ocurrir desde la más amplia y plena participación de las comunidades y actores académicos para garantizar la calidad de los procesos de cambio, de las reformas en la legislación y en las instancias de gobierno colegiadas que hagan posible la generalización de mecanismos de transparencia y comunicación de los programas en marcha, de sus costos y del ejercicio del gasto como un bien público.

En esa perspectiva se han impulsado paradigmas de organización académica, con la creación y desarrollo de unidades inter y transdisciplinarias que permiten la articulación de esfuerzos y la optimización de recursos al trabajar sobre proyectos en red, desde una gran autonomía compartida de sus instan- 
cias orgánicas. Se trata de una nueva manera de operar para mantener activa y dinámica las capacidades anticipatorias de los actores que viven el constante revolucionamiento de sus procesos y resultados científicos y docentes, prever problemas y tendencias de ruptura. Se generan desde estas nuevas instancias orgánicas ambientes institucionales propicios para hacer surgir y proliferar cuerpos académicos híbridos que superan las tradicionales jerarquías y separaciones entre facultades o institutos, la normatividad en extremo detallada y formalizada que entra frecuentemente en contradicción con el carácter esencialmente creativo de la actividad docente e investigativa.

Un salto organizacional central, sin embargo, puede ocurrir si se da inicio a la discusión y propulsión de espacios de articulación de conocimientos y proyectos colaborativos y de su transferencia hacia la sociedad. No es raro en el seno de las universidades encontrar importantes desarrollos en la producción de nuevos conocimientos, pero ahora debe desplegarse una función sustantiva más, que es la de la transferencia constante de conocimientos en propuestas que hagan posible la innovación social.

Una efectiva transferencia de conocimientos depende de la formación y desarrollo de habilidades y aprendizajes específicos, para adaptar el conocimiento producido y transmitido para su uso social y económico. Esto tiene que ver con las perspectivas de responsabilidad y bien público que se organizan desde adentro de la institución hacia afuera en el contexto en el que estos procesos son altamente valorados a nivel del Estado, de las regiones, del país y desde el más amplio espectro de las redes internacionales del conocimiento. Una economía que dinamiza la relación entre conocimiento académico con la innovación social en proyectos estratégicos de amplio impacto hacia el bienestar colectivo, requiere de un cambio institucional que lo haga propicio y lo genere de forma explícita, más allá de las buenas intenciones y de los resultados individuales de los expertos universitarios.

Desde estas bases institucionales que comprenden un sistema complejo de aprendizajes significativos y capacidades orgánicas muy dinámicas se van superando las tradicionales líneas de demarcación y operación de las disciplinas, de los resultados de investigación enmarcados en un Modo 1 de comportamiento lineal, y se van des-formalizando funciones antes constreñidas a las decisiones de arriba hacia abajo, para ir siendo reemplazadas por correlaciones complejas y multivariadas de estructuras y personas que se alteran entre sí en el tiempo y en el espacio. Alrededor de nuevas habilidades y capacidades para articular la producción de un nuevo conocimiento y su transferencia. Cuando las universidades han dinamizado sus estructuras y realizado cambios 
institucionales como los que aquí se proponen, se pueden alcanzar niveles de flexibilidad operacional desde sí misma que permiten conducir de forma más adecuada la manera como ocurren los procesos de innovación desde los nuevos modos de crear conocimiento.

Surge así una nueva política universitaria que opera desde estructuras y procesos horizontales en red, con agendas basadas en desarrollos estratégicos, en prioridades que se dinamizan de manera frecuente, que hace posible una constante articulación de procesos y resultados académicos con los intereses nacionales y los actores involucrados en el mundo del trabajo y de la producción, en breve bajo redes académico-culturales convergentes e interinstitucionales, así como en vínculo estrecho a políticas de desarrollo científico-tecnológicas.

Desde la perspectiva de países como México y otros de América Latina, la propuesta de un modelo de universidad como el que aquí se presenta puede propiciar nuevas estrategias de articulación, integración regional y permitir saltos de calidad en la producción y transferencia de nuevos conocimientos y aprendizajes, y con ello hacer probable una relación con los avances y fronteras del conocimiento que ocurren de manera extraordinaria y simultánea, dinámica y constante en todo el mundo.

Desde esta perspectiva epistémica, el eje de articulación de los procesos académicos se concentra en el logro de objetivos cognitivos y en la gestión de bases de aprendizaje para el descubrimiento, para la resolución de problemas, para la construcción de capacidades de indagación, de creatividad de conceptualización en donde el docente se transforma en un investigador cognitivo y los alumnos concentran su esfuerzo en un aprendizaje significativo de los procesos de descubrimientos de las dinámicas de la naturaleza y de la sociedad.

Este eje central de modelo interno de la universidad haría posible el desarrollo de un modelo de innovación con responsabilidad social, bajo la comprensión de que ello requeriría el establecimiento de cambios importantes en las estructuras básicas y las funciones de las instituciones, sumidas en esquemas tradicionales de oferta de carreras, en un currículum rígido y en niveles d extrema burocratización que ahogan el trabajo académico creativo e innovador.

Desde un enfoque prospectivista, como el que se ha manejado en este trabajo, el objetivo de superación de estas magras condiciones está concentrado en posibilitar una trayectoria de transformación hacia un nuevo modelo que pase de la organización de un sistema escolar a uno de producción y transferencia de conocimientos y aprendizajes. Esta trayectoria o transición de un 
modelo a otro, tiene como imagen el establecimiento de cambios en los aspectos sustantivos de la organización y gestión académico-socio-institucional de la universidad, desde un concepto de calidad como transformación y un paradigma de autonomía como bien público,

Un modelo de universidad de pertinencia e innovación social, hace referencia a una institución activa y dinámica que lidera la transición hacia una sociedad del conocimiento con justicia y bienestar común, una que asume el compromiso y la responsabilidad de asistir e impulsar el cambio social y económico, la democracia, la paz y la sustentabilidad para un desarrollo compartido y equitativo. Es una universidad en donde la calidad del valor de sus conocimientos y aprendizajes se presenta como un principio ordenador de sus capacidades organizativas y el eje de sus cambios, es una universidad mucho más adecuada al periodo en el que se vive de superación de un contexto caracterizado por la injusticia, la ignorancia y el beneficio de los conocimientos, de la ciencia y la tecnología para unos cuantos.

\section{REFERENCIAS}

BAUMAN, Zygmunt. Tiempos líquidos. Argentina: Ensayo Tusquets, 2008. BRENNER, Robert. La economía de la turbulencia global. México: Ed. ERA, 2013.

CUKIER, Kenneth; MAYER-SCHOENBERGER. The Rise of the Big Data. Foreign Affairs, New York , p. 28-40, may-june, 2013.

DIDRIKSSON, Axel. Universidad y sociedades del conocimiento. México: UNESCO-México, 2007.

GIBBONS, Michael et al. La nueva producción del conocimiento. Barcelona: Pomares, 2007.

INNERARITY, Daniel. La democracia del conocimiento, por una sociedad inteligente. Barcelona: Paidós, 2012.

MOLANO, Diego et al. Sociedad de la Información, novedades. Economía Exterior, Madrid, p. 7-105, n. 64, 2013.

STEVENS, Candice. The knowledge driven economy. The knowledge economy. Boston: Butterworth-Heinemann, 1998. 
